\title{
ON THE RELATIONS BETWEEN SOME RATE-OF-GROWTH CONDITIONS
}

\author{
T. G. MCLAUGHLIN
}

\begin{abstract}
We discuss the implications and nonimplications between four rate-of-growth properties of sets useful in certain areas of recursion theory; all nonimplications are established within the boolean algebra generated by the recursively enumerable sets.
\end{abstract}

If $S$ is an infinite set of natural numbers, we denote by $p_{S}$ that strictly increasing function (the so-called principal function of $S$ ) from the set $N$ of all natural numbers into $N$ whose range is $S$. In the present paper, $S$ and $T$ (with or without subscripts) always denote infinite subsets of $N$. For any function $f$, $\delta f$ denotes the domain of $f . \Sigma_{1}^{0}$ denotes the class of all recursively enumerable subsets of $N$, while $\Pi_{1}^{0}$ denotes $\left\{S \mid N-S \in \Sigma_{1}^{0}\right\}$. By a d.r.e. set, we mean one which is the difference of two elements of $\Sigma_{1}^{0}$. S denotes the Turing degree of $S$, and $\bar{S}$ denotes the complement of $S$ in $N$. Let $\left\langle\varphi_{i}\right\rangle$ be some standard recursive enumeration of the partial recursive functions of one argument. As usual, $\mu$ denotes the least number operator.

We wish to catalog the various implications and nonimplications among the universal quantifications of the following four "rate-of-growth" conditions which have been studied in [1], [2], [5], [6], and various other places in the recursion-theoretic literature; in the case of each nonimplication, we shall locate a counterexample within one or another familiar subclass of the $\exists \forall \cap \forall \exists$ level of the arithmetical hierarchy.

$$
\begin{array}{ll}
{\left[\mathbf{D}_{\mathbf{i}}(\mathbf{S})\right]} & (\exists m)(\forall n \geqslant m)\left[n \in \delta \varphi_{i} \Rightarrow p_{S}(n)>\varphi_{i}(n)\right] ; \\
{\left[\mathbf{D}_{1}^{*}(\mathbf{S})\right]} & (\exists m)(\forall n \geqslant m)\left[p_{S}(n) \in \delta \varphi_{i} \Rightarrow p_{S}(n+1)>\varphi_{i}\left(p_{S}(n)\right)\right] ; \\
{\left[\mathbf{D}_{i}^{* *}(\mathbf{S})\right]} & S \subseteq \delta \varphi_{i} \Rightarrow\left[D_{i}^{*}(S)\right] ; \\
{\left[\mathbf{U H}_{i}(\mathbf{S})\right]} & \varphi_{i} \text { total } \Rightarrow\left[D_{i}^{*}(S)\right] .
\end{array}
$$

Let $D(S)$ mean $(\forall i)\left[D_{i}(S)\right]$; similarly for the notations " $D^{*}(S)$ ", " $D^{* *}(S)$ ", " $U H(S)$ ". (We have chosen the notation " $U H$ " since the condition $U H(S)$ has several times been referred to in the literature as uniform hyperimmunity of $S$; in the other cases, " $D$ " is for domination.)

We begin by stating a result from [1] which is just a bit weaker than one of the facts we shall need:

Received by the editors April 6, 1977.

AMS (MOS) subject classifications (1970). Primary 02F99.

Key words and phrases. Retraceable, cohesive, $\Sigma_{1}^{0}$. 
LEMMA 1 (DegTeV). Let $M$ be a maximal $\Sigma_{1}^{0}$ set. Then $U H(\bar{M})$.

(Lemma 1 is also mentioned, but not proved, in [3]. In point of fact, Degtev asserts in [1] that $D^{*}(\bar{M})$ holds; his proof, however, stops just short of showing it, the stopping point being $U H(\bar{M})$. In the next lemma, we carry the matter one easy step further.)

Recall that an infinite set $C \subseteq N$ is called cohesive if there is no set $W \in \Sigma_{1}^{0}$ such that both $C \cap W$ and $C \cap \bar{W}$ are infinite (so that, in particular, maximal $\Sigma_{1}^{0}$ sets are just those which have infinite, cohesive complements).

LEMMA 2. Let $C$ be a cohesive set such that $C \subseteq \bar{M}$ holds for some maximal element of $\Sigma_{1}^{0}$. Then $D^{*}(C)$.

Proof. It is very easily seen that $(\forall S)(\forall T)\left[\left(D^{*}(S) \& T \subseteq S\right) \Rightarrow D^{*}(T)\right]$; hence, it is enough to show that $D^{*}(\bar{M})$ holds. Now, given $\varphi_{i}$, the cohesiveness of $\bar{M}$ implies that either $\bar{M} \cap \delta \varphi_{i}$ or $\bar{M}-\delta \varphi_{i}$ is finite. If $\bar{M} \cap \delta \varphi_{i}$ is finite, then $\left[D_{i}^{*}(\bar{M})\right]$ holds "vacuously". If, on the other hand, $\bar{M}-\delta \varphi_{i}$ is finite, then (by reason of the "Reduction Theorem") there is a total recursive function $\varphi_{j}$ such that $\varphi_{j}$ and $\varphi_{i}$ agree on $\bar{M} \cap \delta \varphi_{i}$. By Lemma $1,\left[D_{j}^{*}(\bar{M})\right]$. Hence, since $\varphi_{j}$ extends $\varphi_{i}$ on $\bar{M},\left[D_{i}^{*}(\bar{M})\right]$. Thus $D^{*}(\bar{M})$, and the lemma is proved.

LEMMA 3 [7]. There exists a maximal $\Sigma_{1}^{0}$ set $M$ such that $\mathbf{M}<\mathbf{0}^{\prime}$.

As we noted at the beginning of the proof of Lemma $2, D^{*}$ is a hereditary property; this is true also of $D$ and $U H$. The situation with respect to $D^{* *}$ is quite different, as our proof of Proposition 6, based on the next lemma, will show. (For background material regarding retraceability, see [2] or [5].)

LEMMA 4. Let $S$ be an infinite retraceable set such that $\neg D^{*}(S)$. Then there is a $\Sigma_{1}^{0}$ set $C$ such that $S \cap C$ is infinite $\& \neg D^{* *}(S \cap C)$.

Proof. Let $g$ be a partial recursive function which retraces $S$, and let $\varphi=\varphi_{i_{0}}$ be such that $\neg\left[D_{i_{0}}^{*}(S)\right]$. We assume, w.l.o.g., that $g(x) \leqslant x$ for all $x \in \delta g$. Let $g^{s}, \varphi^{s}$ denote, respectively, the sets of pairs belonging to $g, \varphi$ after $s$ steps in some fixed recursive enumeration of all pairs in $g, \varphi$ (with exactly one pair entering each of $g, \varphi$ at each step of the enumeration). We shall enumerate $C$, along with a partial recursive function $\Psi$ having domain $C$, in stages, as follows.

Stage 0 . Set $C^{0}=\Psi^{0}=\varnothing$; then proceed to Stage 1 .

Stage $s+1$. For each $x$, let

$$
D_{x}^{s}=\left\{z \mid x \in \delta \varphi^{s}-\delta \Psi^{s} \& x<z \leqslant \varphi^{s}(x) \&\langle z, x\rangle \in g^{s}\right\} .
$$

Let 
$E^{s}=\left\{z \mid(\exists t<s)(\exists x)\left[\langle z, x\rangle \in g^{s}\right.\right.$

$$
\begin{gathered}
\& x \in \delta \varphi^{t}-\delta \Psi^{t} \& D_{x}^{t} \neq \varnothing \& x<z \leqslant \varphi^{t}(x) \\
\left.\left.\&(\forall y<x)\left[y \notin \delta \varphi^{t}-\delta \Psi^{t} \vee D_{y}^{t}=\varnothing\right] \& z \notin C^{s}\right]\right\} .
\end{gathered}
$$

If there is no $x$ such that $x \in \delta \varphi^{s}-\delta \Psi^{s} \& D_{x}^{s} \neq \varnothing$, set $C^{s+1}=C^{s} \cup E^{s}$ and $\Psi^{s+1}=\Psi^{s} \cup\left\{\langle w, 0\rangle \mid w \in E^{s}-\delta \Psi^{s}\right\}$; then proceed to Stage $s+2$. Otherwise, let $x_{0}=(\mu x)\left[x \in \delta \varphi^{s}-\delta \Psi^{s} \& D_{x}^{s} \neq \varnothing\right]$, and define:

Then proceed to Stage $s+2$.

$$
\begin{aligned}
& C_{0}^{s+1}=C^{s} \cup\left\{x_{0}\right\} \cup D_{x_{0}}^{s} ; \\
& \Psi_{0}^{s+1}=\Psi^{s} \cup\left\{\left\langle x_{0}, \varphi^{s}\left(x_{0}\right)\right\rangle\right\} \\
& C^{s+1}=C_{0}^{s+1} \cup E^{s} ; \\
& \Psi^{s+1}=\Psi_{0}^{s+1} \cup\left\{\langle w, 0\rangle \mid w \in C^{s+1}-\delta \Psi_{0}^{s+1}\right\} .
\end{aligned}
$$

We define $C=\cup_{s} C^{s}, \Psi=\cup_{s} \Psi^{s}$. Clearly, $C$ is $\Sigma_{1}^{0}$ and $\Psi$ is a recursively enumerable set of pairs such that $(\forall s)\left[\Psi^{s} \subseteq \Psi^{s+1}\right]$. Since, by a trivial induction on $s$, each $\Psi^{s}$ is seen to be a function, we have that $\Psi$ is a partial recursive function. Obviously $\delta \Psi=C$; moreover, if $x<y \&\{x, y\} \subseteq C \cap$ $S \& g(y)=x \& x=p_{S}(n)$, then $y=p_{S}(n+1)$. The lemma will therefore be proved if we can justify the following claim: there is a sequence $\left\{\left\langle x_{i}, y_{i}\right\rangle\right\}$ of pairs such that $(\forall i)\left[x_{i}<y_{i}<x_{i+1} \&\left\{x_{i}, y_{i}\right\} \subseteq S \cap \delta \Psi \& g\left(y_{i}\right)=x_{i} \&\right.$ $\Psi\left(x_{i}\right) \geqslant y_{i}$. Suppose we have found the first $n_{0}$ terms, $\left\langle x_{0}, y_{0}\right\rangle, \ldots$, $\left\langle x_{n_{0}-1}, y_{n_{0}-1}\right\rangle$, of such a sequence. (If $n_{0}=0$, we are starting from scratch.) Let $s_{0}=(\mu s)$ [all $x_{i}$ and $y_{i}, i\left\langle n_{0}\right.$, belong to $\left.\delta \Psi^{s}\right]$. Let $\langle x, y\rangle$ be the lexicographically least pair such that: $\{x, y\} \subseteq S, n_{0}>0 \Rightarrow x>g(x)>$ $y_{n_{0}-1},\{x, y\} \cap \delta \Psi^{s_{0}}=\varnothing, x<y, g(y)=x, x \in \delta \varphi-\delta \varphi^{s_{0}}$, and $\varphi(x) \geqslant y$. Let $w_{0}=(\mu w)\left[x \in \delta \varphi^{w}\right]$. We claim that $x \in \delta \Psi$. For let $t_{0}=(\mu t)[\{x, y\} \subseteq$ $\left.\delta g^{t}\right]$, and suppose that $x \notin \delta \Psi$. Then, at every stage $t \geqslant \max \left\{w_{0}, t_{0}\right\}$, we have $x \in \delta \varphi^{t}-\delta \Psi^{t} \& D_{x}^{t} \neq \varnothing$. But this clearly yields a contradiction, since then $x$ must enter $\delta \Psi$ no later than during stage $\max \left\{w_{0}, t_{0}\right\}+x_{0}+1$. Thus, $x \in \delta \Psi$. But then, as a trivial induction on $s$ shows, we have either $\Psi(x)=$ $\varphi(x)$ or $\Psi(x)=0$. If $\Psi(x)=\varphi(x)$, let $z_{0}=(\mu z)\left[x \in \delta \Psi^{z}\right]$; then either $y \in$ $\delta \Psi \& \Psi(y)=\varphi(y)$ or else $y$ enters $\delta \Psi$ via membership in $D_{x}^{s} \cup E^{s}$ for some $s \geqslant z_{0}$. But then we can define $x_{n_{0}}=x, y_{n_{0}}=y$. If, on the other hand, $\Psi(x)=0$, then, as is clear from the construction, we must have $g(x) \in \delta \Psi \&$ $\Psi(g(x))=\varphi(g(x)) \geqslant x$; so, in this case, we can define $x_{n_{0}}=g(x), y_{n_{0}}=x$. By induction, then, the required sequence $\left\{\left\langle x_{i}, y_{i}\right\rangle\right\}$ exists and the lemma is proved.

We are now ready to present our "catalog".

Proposition 1. $D^{*}(S) \Rightarrow D^{* *}(S) \Rightarrow U H(S)$.

Proof. Obvious, from definitions.

Proposition 2. If $S$ is regressive, then $D^{*}(S) \Rightarrow D(S)$. 
Proof. It is clear that any regressive set satisfying condition $D^{*}$ is in fact retraceable. Now use [5, proof of Theorem 3.2].

Proposition 2 might seem a bit strange at first sight, since $\left[D_{i}(S)\right]$ involves the action of $\varphi_{i}$ on $\bar{S}$ while $\left[D_{i}^{*}(S)\right]$ does not. The next proposition redresses the intuitive balance.

Proposition 3. There exists a $\Pi_{1}^{0}$ set $S$ such that $D^{*}(S) \& \neg D(S)$.

Proof. As shown in [8], $D(S) \Rightarrow \mathbf{S}>0^{\prime}$. Applying Lemma 3 , let $S$ be a cohesive $\Pi_{1}^{0}$ set such that $S<\boldsymbol{\sigma}$. Then $\neg D(S)$. On the other hand, $D^{*}(S)$ holds by Lemma 2 .

Proposition 4. There exists a retraceable $\Pi_{1}^{0}$ set $S$ such that $D(S) \&$ $\neg U H(S)$.

Proof. By (for instance) [5, Theorems 3.1 and 3.2], there is a retraceable $\Pi_{1}^{0}$ set $T$ such that $D(T)$ holds. By [6, Theorem 4.1], there is a second retraceable $\Pi_{1}^{0}$ set $R$ such that $p_{R} \circ p_{T}$ is the principal function of a set $S$ for which $\neg U H(S)$. But, the condition $D$ is (as is very easily seen) preserved under compositional injection; and, the composition of principal functions of two infinite retraceable $\Pi_{1}^{0}$ sets is again an infinite retraceable $\Pi_{1}^{0}$ set. $S$ therefore verifies our proposition. (Easy direct constructions also are available for proving Proposition 4.)

Proposition 5. There exists a retraceable $\Pi_{1}^{0}$ set $S$ such that $D^{* *}(S) \&$ $\neg D^{*}(S)$.

Proof. By [2], [4], and [7], let $S$ be a retraceable $\Pi_{1}^{0}$ set such that $S<\sigma \quad \boldsymbol{\sigma}$ $D^{* *}(S)$. By [8] plus Proposition 2, we have $\neg D^{*}(S)$.

Proposition 6. There exists an infinite d.r.e. set $S$ such that $U H(S) \&$ $\neg D^{* *}(S)$.

Proof. Applying Proposition 5, let $S_{0}$ be a retraceable $\Pi_{1}^{0}$ set such that $\neg D^{*}\left(S_{0}\right) \& D^{* *}\left(S_{0}\right)$. Applying Lemma 4 , let $C$ be a $\Sigma_{1}^{0}$ set such that $S_{0} \cap C$ is infinite $\& \neg D^{* *}\left(S_{0} \cap C\right)$. Since $U H$ is a hereditary condition, and since $U H$ and $D^{* *}$ are equivalent for $\Pi_{1}^{0}$ sets (using the "Reduction Theorem"), we see that $S=S_{0} \cap C$ verifies the proposition.

Several fairly obvious questions occur in connection with the foregoing results:

QI. Is there a cohesive set $C$ such that $\neg D^{*}(C)$ ?

QII. Is there a complete maximal $\Sigma_{1}^{0}$ set $M$ such that $\neg D(\bar{M})$ ?

QIII. If $S$ is $\Pi_{1}^{0}$, can the set $S \cap C$ of Lemma 4 (and hence the set $S$ of Proposition 6) be required to be retraceable (or even, merely, regressive)? In an earlier version of this paper, we claimed this could be done. The referee, however, spotted a formidable gap in the proof; retraceability was lost during repairs.

QIV. Is Lemma 4 a nonvacuous assertion? That is, is there an example of 
an infinite set $S$ such that $\neg D^{*}(S) \&\left(\forall C \in \Sigma_{1}^{g}\right)\left[S \cap C\right.$ infinite $\Rightarrow D^{* *}(S \cap$ C)]?

\section{REFERENCES}

1. A. N. Degtev, Hypersimple sets with retraceable complements, Algebra i Logika 10 (1971), 235-246. (Russian) MR 44 \#5221.

2. E. Ellentuck, On the degrees of universal regressive isols, Math. Scand. 32 (1973), 145-164. MR 48 \# 10789.

3. H. Gonshor, Recursive density types. II, Trans. Amer. Math. Soc. 140 (1969), 505-509. MR 40 \#4102.

4. D. A. Martin, Classes of recursively enumerable sets and degrees of unsolvability, Z. Math. Logik Grundlagen Math. 12 (1966), 295-310. MR 37 \#68.

5. T. G. McLaughlin, Rees and isols. I, Rocky Mountain J. Math. 5 (1975), 401-418. MR 54 \#2438.

6. __ Trees and isols. II, Z. Math. Logik Grundlagen Math. 22 (1976), 45-78.

7. G. E. Sacks, A maximal set which is not complete, Michigan Math. J. 11 (1964), 193-205. MR 29 \#3368.

8. S. Tennenbaum, Degree of unsolvability and the rate of growth of functions, Proc. Sympos. Math. Theory of Automata, New York, 1962, 71-73. MR 29 \#4679.

Department of Mathrmatics, TeXas Tech University, Lubbock, Texas 79409 